\title{
Risk factors, aetiology and outcome of ischaemic stroke in young adults: the Swiss Young Stroke Study (SYSS)
}

\author{
Barbara Goeggel Simonetti ${ }^{1}$ Marie-Luise Mono ${ }^{1}$ Uyen Huynh-Do ${ }^{2}$ • \\ Patrik Michel $^{3}$ - Celine Odier ${ }^{3} \cdot$ Roman Sztajzel $^{4} \cdot$ Philippe Lyrer $^{5}$. \\ Stefan T. Engelter ${ }^{5}$ Leo Bonati ${ }^{5} \cdot$ Henrik Gensicke $^{5}$ - Christopher Traenka ${ }^{5}$. \\ Barbara Tettenborn $^{6} \cdot$ Bruno Weder $^{6} \cdot$ Urs Fischer $^{1} \cdot$ Aekaterini Galimanis $^{1}$ • \\ Simon Jung ${ }^{1}$. Rudolf Luedi ${ }^{1}$ - Gian Marco De Marchis ${ }^{1}$ Anja Weck ${ }^{1}$. \\ Carlo W. Cereda ${ }^{7} \cdot$ Ralf Baumgartner $^{8}$ - Claudio L. Bassetti ${ }^{1} \cdot$ Heinrich P. Mattle $^{1}$. \\ Krassen Nedeltchev $^{1,9} \cdot$ Marcel Arnold ${ }^{1}$
}

Received: 15 February 2015/Revised: 26 May 2015/Accepted: 28 May 2015/Published online: 12 June 2015 (c) Springer-Verlag Berlin Heidelberg 2015

\begin{abstract}
Ischaemic stroke (IS) in young adults has been increasingly recognized as a serious health condition. Stroke aetiology is different in young adults than in the older population. This study aimed to investigate aetiology and risk factors, and to search for predictors of outcome and recurrence in young IS patients. We conducted a prospective multicentre study of consecutive IS patients aged 16-55 years. Baseline demographic data, risk factors, stroke aetiology including systematic genetic screening for Fabry disease and severity were assessed and related to functional neurological outcome (modified Rankin Scale, $\mathrm{mRS}$ ), case fatality, employment status, place of residence, and recurrent cerebrovascular events at 3 months. In 624 IS patients (60\% men), median age was 46 (IQR 39-51) years and median NIHSS on admission 3 (IQR 1-8).
\end{abstract}

B. Goeggel Simonetti and M.-L. Mono contributed equally.

K. Nedeltchev and M. Arnold jointly directed this work.

Electronic supplementary material The online version of this article (doi:10.1007/s00415-015-7805-5) contains supplementary material, which is available to authorized users.

Marcel Arnold

marcel.arnold@insel.ch

Barbara Goeggel Simonetti

barbara.goeggelsimonetti@insel.ch

1 Department of Neurology, Inselspital, University Hospital, University of Bern, Freiburgstrasse 10, 3010 Bern, Switzerland

2 Department of Nephrology, Inselspital, University Hospital, University of Bern, Bern, Switzerland

3 Neurology Service, Centre Hospitalier Universitaire Vaudois, Lausanne University, Lausanne, Switzerland
Modifiable vascular risk factors were found in $73 \%$. Stroke aetiology was mostly cardioembolism (32\%) and of other defined origin (24\%), including cervicocerebral artery dissection (17\%). Fabry disease was diagnosed in 2 patients $(0.3 \%)$. Aetiology remained unknown in $20 \%$. Outcome at 3 months was favourable (mRS 0-1) in $61 \%$ and fatal in $2.9 \%$. Stroke severity $(p<0.001)$ and diabetes mellitus $(p=0.023)$ predicted unfavourable outcome. Stroke recurrence rate at 3 months was $2.7 \%$. Previous stroke or TIA predicted recurrent cerebrovascular events $(p=0.012)$. In conclusion, most young adults with IS had modifiable vascular risk factors, emphasizing the importance of prevention strategies. Outcome was unfavourable in more than a third of patients and was associated with initial stroke severity and diabetes mellitus. Previous cerebrovascular events predicted recurrent ones.

Keywords Ischaemic stroke - Stroke in young adults . Risk factors - Aetiology $\cdot$ Stroke recurrence $\cdot$ Outcome

4 Department of Neurology, University Hospital Geneva, Geneva, Switzerland

5 Department of Neurology, University Hospital Basel, Basel, Switzerland

6 Department of Neurology, Cantonal Hospital St.Gallen, St.Gallen, Switzerland

7 Department of Neurology, Neurocenter (EOC) of Southern Switzerland, Ospedale Civico, Lugano, Switzerland

8 NeuroCenter, Hirslanden Klinik, Zurich, Switzerland

9 Department of Neurology, Cantonal Hospital Aargau, Aarau, Switzerland 


\section{Introduction}

Ischaemic stroke (IS) in young adults and adolescents has been considered uncommon. However, incidence is estimated around 10 per 100,000 young adults per year [1, 2]. With the rising number of publications on stroke in young adults, the index of suspicion, diagnosis and finally incidence of the condition may increase. Previous research showed a broader spectrum of aetiologies and potentially treatable causes compared to older stroke patients, and a high percentage of unknown stroke aetiologies in young adults [1-9]. Research on outcome of young adults after IS is largely limited to single centre, some of them retrospective studies, showing residual physical and psychosocial impairment in at least a third of patients $[3,6-8,10$ 12]. Apart from the potentially deleterious consequences for the individual patient, stroke in young adults has a considerable socioeconomic impact because of loss of working years and long-term dependency on nursing and social care. Thus, stroke prevention and improvement of outcome are particularly important in this group. In order to tailor better preventive strategies and to improve outcome of young IS patients, we still need more information not only on stroke risk factors and aetiology, but also on predictors of recurrent cerebrovascular events and outcome. The aim of this study was not only to prospectively and thoroughly investigate risk factors and aetiologies of IS in young adults, attempting to minimize the rate of unknown aetiologies, but also to assess predictors of clinical outcome and recurrent cerebrovascular events at 3 months.

\section{Methods}

The Swiss Young Stroke Study (SYSS) is a prospective multicentre study enrolling consecutive patients aged 16-55 years directly admitted to one of the University Hospitals of Basel, Bern, Geneva, Lausanne or the Cantonal Hospital of St. Gallen from 07/2008 until 01/2012 for IS (both first-ever-in-a-lifetime stroke, FELS, and recurrent events).

The local ethical review committees at all study sites approved the study. Written informed consent was obtained from all patients or their next-on-kin.

Exclusion criteria were intracranial haemorrhage on CT scan or MRI and all other disorders that presented with an acute focal neurological deficit and were not ischaemic in origin.

Patients underwent a comprehensive diagnostic stroke workup, including a clinical neurological examination and scoring with the National Institutes of Health Stroke Scale
(NIHSS) on admission [13], laboratory examination, brain and neurovascular imaging, and in all patients except of those with a clear non-cardioembolic cause of stroke, transoesophageal echocardiography, and 24-h electrocardiogram (ECG) to determine stroke aetiology using the Trial of Org 10172 in Acute Stroke Treatment (TOAST) criteria [14]. The following stroke risk factors were assessed: family history of IS (defined as IS in first degree relatives), personal history of previous IS (defined as radiological signs of a non-acute IS on brain imaging with or without symptoms of IS) or previous transient ischaemic attack (TIA, defined as a neurological deficit lasting $<24 \mathrm{~h}$ ), atrial fibrillation (diagnosed on an ECG), coronary heart disease, and the modifiable vascular risk factors hypercholesterolemia (total venous plasma cholesterol $\geq 5 \mathrm{mmol} / 1$ or use of cholesterol-lowering medication), current smoking or cessation of smoking in the 10 years prior to IS, diabetes mellitus (defined as two fasting venous plasma glucose values $\geq 7 \mathrm{mmol} / \mathrm{l}$ or use of antidiabetic medication), hypertension (defined by preadmission history or use of antihypertensive medication), oestrogen substitution in women (oral contraceptives or hormone replacement therapy). In addition, patients were routinely screened for Fabry disease and, if appropriate, for blood coagulation disorders. Systematic genetic screening for Fabry disease consisted in complete sequencing of the gene encoding alpha-galactosidase A (GLA; gene MIM number 300644) in genomic DNA from patients' leukocytes. In equivocal cases, determination of blood globotriaosylceramide $\mathrm{Gb} 3$ (norm $<4 \mu \mathrm{g} / \mathrm{ml}$ ) and urine $\mathrm{Gb} 3$ (total $\mathrm{Gb} 3$ and $\mathrm{Gb} 3-\mathrm{C} 24$, norm $<35 \mathrm{ng} / \mathrm{mg}$ creatinine) was performed. All analyses were performed at the AlbrechtKossel-Institute, Medical Faculty of the University of Rostock, by staff blinded to the clinical data.

The follow-up examination at 3 months assessed the modified Rankin Scale (mRS) score [15], occupational status, and recurrent cerebrovascular event (TIA/IS). If stroke recurrence was suspected, a CT scan or, if necessary, an MRI was performed. Favourable outcome was defined as an mRS-score of $0-1$.

Statistical analysis was performed using IBM SPSS ${ }^{\circledR}$ version 21. Differences between groups were assessed using Fisher's exact test or Pearson Chi-square test, as appropriate (for categorical variables), and Mann-Whitney $U$ test (for continuous variables). Possible predictors of outcome, recurrence, and survival, that had a regression coefficient $p<0.2$ in the binary logistic regression analysis, were included in a multivariable logistic regression analysis. A probability $(p)$ value $<0.05$ was considered statistically significant. Two-tailed $p$ values are indicated. Subgroup analyses were performed for patients aged 16-45 years compared to patients aged 46-55 years, 
because many previous studies on young stroke included patients up to the age of 45 years only, while studies analysing Fabry disease in young stroke patients included patients up to 55 years.

\section{Results}

\section{Demographics and baseline data}

624 patients $($ men $=375 / 60 \%)$ with IS were included in this analysis. Median age was 46 (IQR 39-51) years, and median NIHSS score on admission 3 (IQR 1-8). A total of 539 of 624 patients with IS (86 \%) had an FELS. Thrombolysis was performed in 158/25 \% (94/15 \% intravenous, 26/4.2 \% intra-arterial, 21/3.4\% bridging), mechanical thrombectomy in $17 / 2.7 \%$ patients.

\section{Risk factors and aetiology}

Ninety-six percent of patients had at least one vascular risk factor, and $73 \%$ at least one modifiable vascular risk factor (Table 1). Most common were hypercholesterolemia (47\%), smoking (43\%), and hypertension (35\%). Fortysix women (19\% of all female patients) were taking oral contraceptives and three $(1.2 \%)$ hormone replacement therapy.

Stroke aetiology was cardioembolism in $32 \%$, of other determined origin in $24 \%$ (of them, 106 patients with cervical artery dissection, 30 with thrombophilia, and 6 with vasculitis), large-artery atherosclerosis in $11 \%$, small-vessel disease in $9 \%$, undetermined despite complete investigation in $16 \%$, undetermined and incompletely investigated in $4.3 \%$ or due to more than one potential pathomechanisms in $4.5 \%$. Screening for Fabry disease was positive in two patients $(0.3 \%$, Table 2$)$.

\section{Outcome}

Three-month follow-up data were obtained in 614 (98\%) patients [clinical visit in $450(73 \%)$, telephone interview in $164(27 \%)$ ]. Outcome at 3 months was favourable (mRS 0-1) in $61 \%$. Eighteen patients had died $(2.9 \%)$. The distribution of all mRS-scores is displayed in Fig. 1.

Information on the patients' place of residence was available in $80 \%$ and on occupational status in $78 \%$ surviving patients. Most patients were living at home (405/ $81 \%)$ and were employed and working (261/56 \%), 149 of them $(24 \%)$ fulltime.

Sixteen $(2.7 \%)$ patients had a recurrent cerebrovascular event (1.2\% IS, $1.5 \%$ TIA).

\section{Predictors of outcome and recurrence}

Diabetes mellitus $(p=0.023, \quad$ OR $0.34,95 \%$ CI $0.13-0.86)$ and NIHSS on admission $(p<0.001$, OR 0.8 , $95 \%$ CI $0.75-0.85$ ) were independent predictors of outcome. The multivariable logistic regression analyses further identified NIHSS on admission as an independent predictor of survival $(p=0.044$, OR $0.91,95 \%$ CI $0.83-0.99)$. Previous stroke or TIA was the only variable significantly predicting recurrence of stroke or TIA $(p=0.012$, OR $3.5,95 \%$ CI $1.32-9.29)$.

These predictors of outcome and recurrence were not significantly different in the 10 patients lost to follow-up. Thus, it is unlikely that the overall results had changed substantially had the missing 10 patients been included into follow-up.

Table 1 Vascular risk factors in the 16-45 and 46-55 years age groups

\begin{tabular}{|c|c|c|c|c|}
\hline Variable (valid $n / \%)$ & $\begin{array}{l}\text { Frequency } \\
(n / \%)\end{array}$ & $\begin{array}{l}\text { Frequency }(n / \%) \text { in } 16-45 \\
\text { years age group }(n=284)\end{array}$ & $\begin{array}{l}\text { Frequency }(n / \%) \text { in } 46-55 \\
\text { years age group }(n=340)\end{array}$ & $p$ \\
\hline Hypercholesterolemia (614/98) & $290 / 47$ & $99 / 35$ & $191 / 58$ & $<0.001$ \\
\hline Smoking (616/99) & $265 / 43$ & $114 / 41$ & $151 / 45$ & 0.288 \\
\hline Hypertension (622/99.7) & $215 / 35$ & $60 / 21$ & $155 / 46$ & $<0.001$ \\
\hline Prior stroke or TIA $(624 / 100)$ & $85 / 14$ & $38 / 13$ & $47 / 14$ & 0.907 \\
\hline Diabetes $(622 / 99.7)$ & $52 / 8$ & $7 / 2.5$ & $45 / 13$ & $<0.001$ \\
\hline Coronary heart disease $(620 / 99)$ & $33 / 5$ & $7 / 2.5$ & $26 / 8$ & 0.004 \\
\hline Atrial fibrillation (606/97) & $22 / 4$ & $5 / 1.8$ & $17 / 5$ & 0.029 \\
\hline Thrombophilia $(376 / 60)$ & $30 / 8$ & $22 / 12$ & $8 / 4.3$ & 0.012 \\
\hline Women with oestrogen intake $(246 / 249)$ & $49 / 20$ & $44 / 32$ & $5 / 4.6$ & \\
\hline Oral contraceptives & & $42 / 31$ & $4 / 3.7$ & $<0.001$ \\
\hline Hormone replacement therapy & & $2 / 1.5$ & $1 / 0.9$ & \\
\hline Family history of stroke (553/87) & $134 / 24$ & $45 / 18$ & $89 / 30$ & 0.002 \\
\hline
\end{tabular}



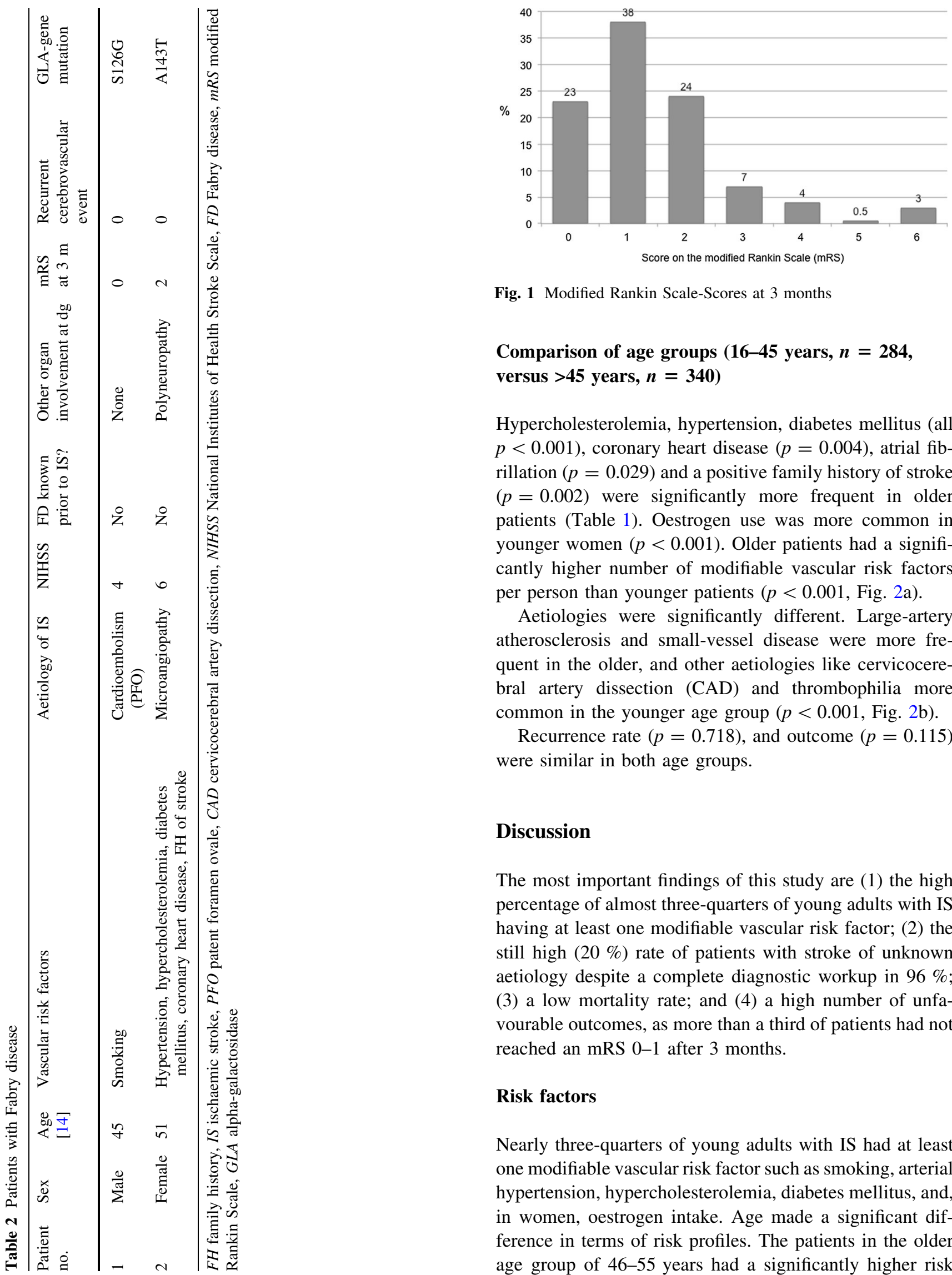

Fig. 1 Modified Rankin Scale-Scores at 3 months

Comparison of age groups (16-45 years, $n=284$, versus $>45$ years, $n=340$ )

Hypercholesterolemia, hypertension, diabetes mellitus (all $p<0.001)$, coronary heart disease $(p=0.004)$, atrial fibrillation $(p=0.029)$ and a positive family history of stroke $(p=0.002)$ were significantly more frequent in older patients (Table 1). Oestrogen use was more common in younger women $(p<0.001)$. Older patients had a significantly higher number of modifiable vascular risk factors per person than younger patients ( $p<0.001$, Fig. 2a).

Aetiologies were significantly different. Large-artery atherosclerosis and small-vessel disease were more frequent in the older, and other aetiologies like cervicocerebral artery dissection (CAD) and thrombophilia more common in the younger age group ( $p<0.001$, Fig. $2 b)$.

Recurrence rate $(p=0.718)$, and outcome $(p=0.115)$ were similar in both age groups.

\section{Discussion}

The most important findings of this study are (1) the high percentage of almost three-quarters of young adults with IS having at least one modifiable vascular risk factor; (2) the still high (20\%) rate of patients with stroke of unknown aetiology despite a complete diagnostic workup in $96 \%$; (3) a low mortality rate; and (4) a high number of unfavourable outcomes, as more than a third of patients had not reached an mRS 0-1 after 3 months.

\section{Risk factors}

Nearly three-quarters of young adults with IS had at least one modifiable vascular risk factor such as smoking, arterial hypertension, hypercholesterolemia, diabetes mellitus, and, in women, oestrogen intake. Age made a significant difference in terms of risk profiles. The patients in the older age group of 46-55 years had a significantly higher risk 
Fig. 2 a Number of modifiable risk factors and $\mathbf{b}$ distribution of aetiologies according to age group. $L A A$ large-artery atherosclerosis, $C E$ cardioembolism, SVD smallvessel disease, $N E$ negative evaluation, $I E$ incomplete evaluation, Multiple more than one aetiology possible
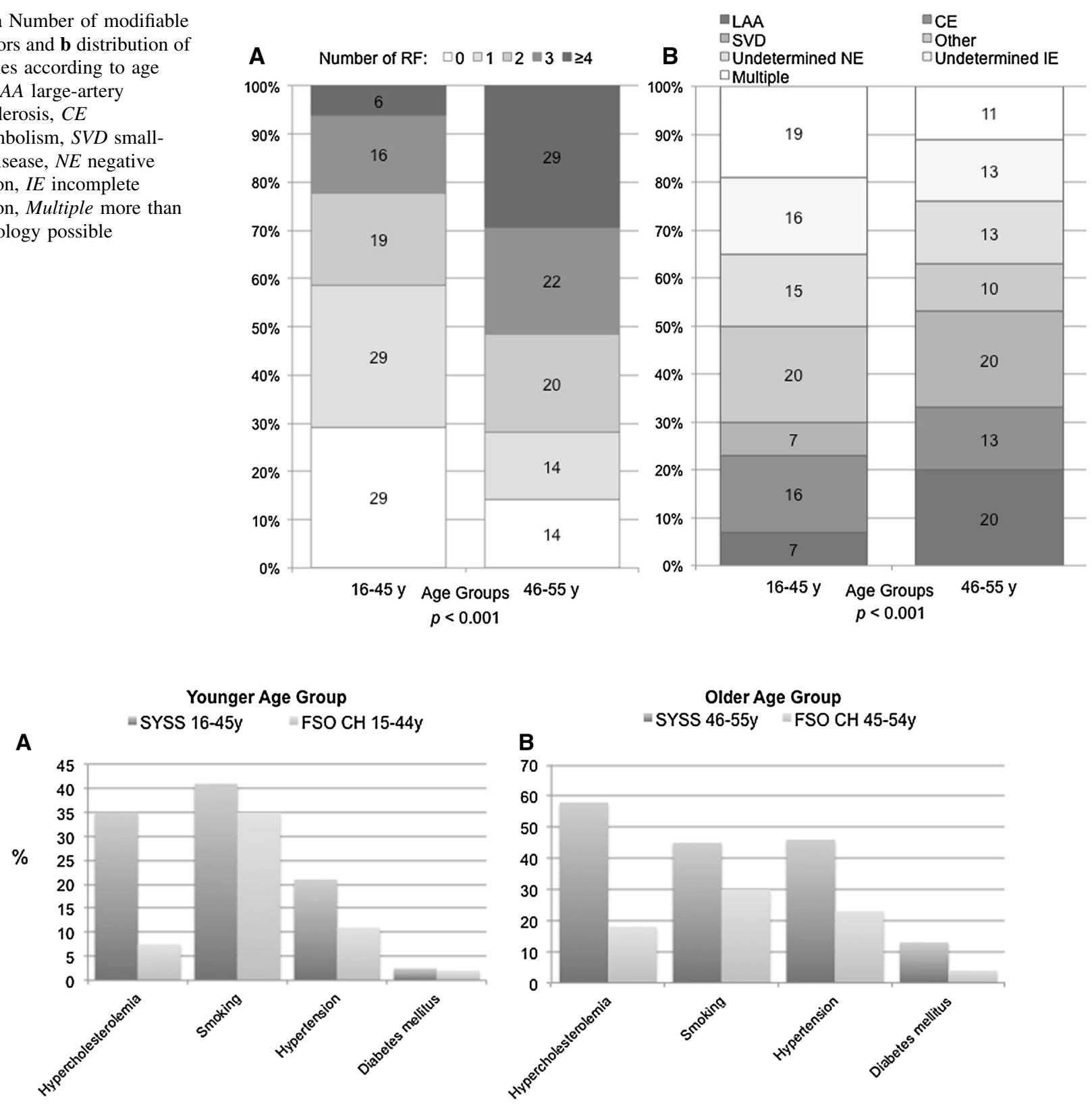

C

Estrogen intake in the general female population, SYSS all females and divided by age groups

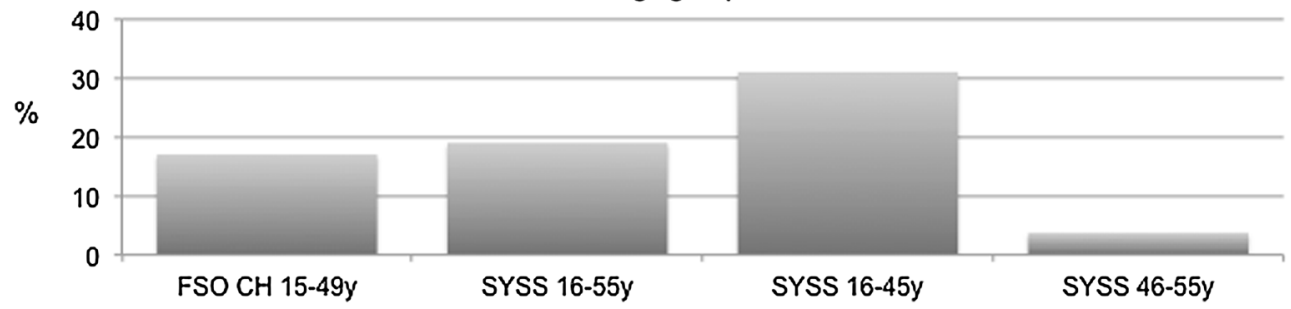

Fig. 3 Modifiable vascular risk factors in young adults with IS compared to the general population. FSO CH Swiss Federal Statistical Office

load compared with adults aged $\leq 45$ years (Table 1 ; Fig. 2a). In both age groups, risk factors were clearly more frequent than in the general population (Fig. 3, based on the
Swiss Health Survey 2012 [16]), taking into account the temporal trends since 1997, thus covering the same time period as the SYSS recruitment phase. 
The high percentage of risk factors and its increase with age is similar to previous research in young adults with stroke $[12,17,18]$.

\section{Aetiology}

With the exception of a small single-centre retrospective study [19], previous studies reported an undetermined IS aetiology in a third up to half of young stroke patients (Suppl Fig. 1) [1-9]. Cardioembolic IS was the most frequent TOAST-subtype in our study, while most previous studies reported cardioembolism in less than a fifth of young patients [1-9]. This difference might be explained by the common use of transoesophageal echocardiography in our study centres, leading to a more frequent detection of cardiac sources of emboli.

Other determined aetiologies, particularly cervical artery dissection (CAD), were the second most frequent aetiology $(17 \%)$, which is in line with previous singlecentre studies $[2,8]$. However, the "sifap" study reported CAD in only $9 \%$ of young IS patients [4]. Again, technical diagnostic aspects such as the routine use of vessel imaging and the use of MRI with fat suppression technique whenever CAD was suspected in our study centres, might explain this difference. Unlike some previous studies demonstrating Fabry disease in up to $4 \%$ of patients with cryptogenic stroke, but in line with the recent prospective "sifap" study, our percentage of patients with Fabry disease was low $(0.3 \%)$ [4, 20-25]. In contrast to sifap, we did not identify any case of "probable" Fabry disease, defined either as the D313Y mutation or a complex intronic haplotype in combination with increase of blood and urine Gb3. Both SYSS patients with Fabry disease were 45 years of age or older at time of diagnosis, thus might have been missed in studies investigating adults up to 45 years only.

Intracranial large-artery atherosclerosis and small-artery disease were the third and fourth most frequent aetiologies, which is in line with the high rate of modifiable risk factors in young stroke patients, emphasizing the importance of primary vascular risk prevention already at young age.

Even though our diagnostic workup was extensive, additional investigations, such as long-term cardiac rhythm monitoring searching for atrial fibrillation, as suggested in the latest literature [26], might further decrease the number of patients with unknown IS aetiology.

\section{Outcome}

Outcome in young adults has been investigated in studies mostly comprising limited patient numbers [1, 3, 6-8, 1012, 27, 28]. Direct comparison of the results is hampered by the differences in outcome measures and timing of follow-up.

Case fatality rates were higher in studies published before 2000 than thereafter [1, 3, 6-8, 27, 28]. After 2000, fatality was generally less than $5 \%$ during the first months after stroke and similar to our fatality rate $(2.9 \%)$.

Previous studies reporting functional outcome with detailed mRS-scores showed mRS 0-1 in 63-71\% and mRS 0-2 in 84-94\% of survivors [3, 6-8, 11, 28], which is in line with our study (mRS 0-1 in 63\%, mRS 0-2 in $88 \%$ of survivors). Even though nearly two-thirds of our patients had a favourable outcome (mRS 0-1), only half of these were working fulltime at 3 months. Functional outcome has not improved over time since the first of the abovementioned studies were published in the late 1990s, despite the decrease of unknown aetiologies. In SYSS, stroke aetiology had no significant impact on outcome.

Few studies investigated the influence of risk factors on recurrence and outcome in young adults with stroke. In line with a previous study of our group on a different population of young adults with stroke, previous IS/TIA was a risk factor for recurrence, and diabetes mellitus and high NIHSS score on admission predictors of unfavourable outcome [6]. In patients with FELS included in the Helsinki Young Stroke Registry, diabetes mellitus was again found to be a risk factor for recurrent IS and other vascular events [29]. Our study did not confirm further findings of the aforementioned and other previous studies, describing the presence of other vascular risk factors than diabetes, vasculopathic and cardioembolic stroke aetiology, older age, or male sex as predictors of recurrence $[6,7,12,30,31]$ and older age, male sex, or the presence of other vascular risk factors as predictors of unfavourable outcome [6-8].

\section{Limitations}

This study is not population based. However, a selection bias due to recruitment in stroke centres is likely to be low, since in Switzerland stroke patients, particularly the young, are commonly admitted to a stroke centre such as the ones participating in SYSS.

When comparing frequencies of vascular risk factors in SYSS-participants with the general population, we have to bear in mind the methodological differences of the Swiss Health Survey and SYSS. The Health Survey data were acquired by a questionnaire survey of a general population sample, asking for hypertension or high cholesterol levels once in life, or diagnosis of diabetes. Assuming that only a minority of young adults in the general population is checked for these conditions, their prevalence is likely to be underestimated. Different definitions of age groups may hamper the comparison of prevalence further. 
We did not assess baseline mRS-scores. However, disability before the stroke is rare in young stroke patients.

The diagnostic workup was broad, but screening for coagulation disorders was only performed in selected patients. Considering the low diagnostic yield of blood coagulation disorder screening in all young patients with IS, the probability of having missed a substantial number of positive results is low. Drug screenings were not performed on a regular basis either.

\section{Conclusions}

To our knowledge, this is the first prospective multicentre study of consecutive ischaemic stroke patients aged 16-55 years that not only provides information on risk factors and stroke aetiology, including rare diseases such as Fabry disease, but also on stroke recurrence and clinical outcome of young stroke patients and the predictors of recurrence and outcome. Most young adults with IS have modifiable vascular risk factors, emphasizing the need for better primary prevention strategies addressing young adults, adolescents and even children. This holds even more true when considering the high morbidity of IS in the young, which leads not only to potentially deleterious consequences for the individual patient, but has a considerable socioeconomic impact due to the reduction in working lifetime and long duration of dependency on nursing and social care. With a thorough diagnostic workup, aetiology can be determined in $80 \%$. Outcome is favourable in less than twothirds and depends on the initial stroke severity (NIHSS) and the presence of diabetes mellitus.

\begin{abstract}
Acknowledgments We thank the staff and participants of all SYSScentres for their important contribution, particularly Marianne Kormann, Andrea Surtmann Huguenin, Niklaus Meier, Mirjam R. Heldner, Oliver Findling, Fabienne Aregger, Ashraf Eskandari and Fabienne Kormann for their help in collecting the data, and Pietro Ballinari for his statistical advice. This study was supported by an unrestricted grant of Shire Human Genetic Therapies ${ }^{\circledR}$, Grand-Rue, 92/CP 1526, 1820 Montreux VD, Switzerland. The Swiss National Science Foundation supported B.G.S., S.J., and U.F. (SNSF SPUM Grants 140340, 33CM30).
\end{abstract}

Conflicts of interest The authors declare no financial or other conflicts of interest.

\section{References}

1. Jacobs BS, Boden-Albala B, Lin IF, Sacco RL (2002) Stroke in the young in the northern Manhattan stroke study. Stroke 33:2789-2793

2. Putaala J, Metso AJ, Metso TM, Konkola N, Kraemer Y, Haapaniemi E et al (2009) Analysis of 1008 consecutive patients aged 15-49 with first-ever ischemic stroke: the Helsinki young stroke registry. Stroke 40:1195-1203

3. Synhaeve NE, Arntz RM, Maaijwee NA, Rutten-Jacobs LC, Schoonderwaldt HC, Dorresteijn LD et al (2014) Poor long-term functional outcome after stroke among adults aged 18-50 years: follow-up of transient ischemic attack and stroke patients and unelucidated risk factor evaluation (FUTURE) study. Stroke 45:1157-1160

4. Rolfs A, Fazekas F, Grittner U, Dichgans M, Martus P, Holzhausen $\mathrm{M}$ et al (2013) Acute cerebrovascular disease in the young: the stroke in young fabry patients study. Stroke 44:340-349

5. Yesilot Barlas N, Putaala J, Waje-Andreassen U, Vassilopoulou S, Nardi K, Odier C et al (2013) Etiology of first-ever ischaemic stroke in European young adults: the 15 cities young stroke study. Eur J Neurol 20:1431-1439

6. Nedeltchev K, der Maur TA, Georgiadis D, Arnold M, Caso V, Mattle HP et al (2005) Ischaemic stroke in young adults: predictors of outcome and recurrence. J Neurol Neurosurg Psychiatry $76: 191-195$

7. Varona JF, Bermejo F, Guerra JM, Molina JA (2004) Long-term prognosis of ischemic stroke in young adults. Study of 272 cases. J Neurol 251:1507-1514

8. Leys D, Bandu L, Henon H, Lucas C, Mounier-Vehier F, Rondepierre $\mathrm{P}$ et al (2002) Clinical outcome in 287 consecutive young adults (15-45 years) with ischemic stroke. Neurology 59:26-33

9. Adams HP Jr, Kappelle LJ, Biller J, Gordon DL, Love BB, Gomez F et al (1995) Ischemic stroke in young adults. Experience in 329 patients enrolled in the Iowa Registry of stroke in young adults. Arch Neurol 52:491-495

10. Rozenthul-Sorokin N, Ronen R, Tamir A, Geva H, Eldar R (1996) Stroke in the young in Israel. Incidence and outcomes. Stroke 27:838-841

11. Waje-Andreassen U, Thomassen L, Jusufovic M, Power KN, Eide GE, Vedeler CA et al (2013) Ischaemic stroke at a young age is a serious event-final results of a population-based longterm follow-up in Western Norway. Eur J Neurol 20:818-823

12. Putaala J, Haapaniemi E, Kaste M, Tatlisumak T (2012) How does number of risk factors affect prognosis in young patients with ischemic stroke? Stroke 43:356-361

13. Brott T, Adams HP Jr, Olinger CP, Marler JR, Barsan WG, Biller $\mathrm{J}$ et al (1989) Measurements of acute cerebral infarction: a clinical examination scale. Stroke 20:864-870

14. Adams HP Jr, Bendixen BH, Kappelle LJ, Biller J, Love BB, Gordon DL et al (1993) Classification of subtype of acute ischemic stroke. Definitions for use in a multicenter clinical trial. TOAST. Trial of Org 10172 in Acute Stroke Treatment. Stroke 24:35-41

15. van Swieten JC, Koudstaal PJ, Visser MC, Schouten HJ, van Gijn J (1988) Interobserver agreement for the assessment of handicap in stroke patients. Stroke 19:604-607

16. Storni MKM, Lieberherr R (2013) Swiss Health Survey 2012. Federal Statistical Office, Neuchâtel, p 32

17. Putaala J, Yesilot N, Waje-Andreassen U, Pitkaniemi J, Vassilopoulou S, Nardi K et al (2012) Demographic and geographic vascular risk factor differences in European young adults with ischemic stroke: the 15 cities young stroke study. Stroke 43:2624-2630

18. von Sarnowski B, Putaala J, Grittner U, Gaertner B, Schminke U, Curtze S et al (2013) Lifestyle risk factors for ischemic stroke and transient ischemic attack in young adults in the stroke in young fabry patients study. Stroke 44:119-125

19. Ji R, Schwamm LH, Pervez MA, Singhal AB (2013) Ischemic stroke and transient ischemic attack in young adults: risk factors, diagnostic yield, neuroimaging, and thrombolysis. JAMA Neurol 70:51-57 
20. Baptista MV, Ferreira S, Pinho EMT, Carvalho M, Cruz VT, Carmona $\mathrm{C}$ et al (2010) Mutations of the GLA gene in young patients with stroke: the PORTYSTROKE study-screening genetic conditions in Portuguese young stroke patients. Stroke 41:431-436

21. Brouns R, Sheorajpanday R, Braxel E, Eyskens F, Baker R, Hughes D et al (2007) Middelheim Fabry Study (MiFaS): a retrospective Belgian study on the prevalence of Fabry disease in young patients with cryptogenic stroke. Clin Neurol Neurosurg 109:479-484

22. Brouns R, Thijs V, Eyskens F, Van den Broeck M, Belachew S, Van Broeckhoven C et al (2010) Belgian Fabry study: prevalence of Fabry disease in a cohort of 1000 young patients with cerebrovascular disease. Stroke 41:863-868

23. Rolfs A, Bottcher T, Zschiesche M, Morris P, Winchester B, Bauer P et al (2005) Prevalence of Fabry disease in patients with cryptogenic stroke: a prospective study. Lancet 366:1794-1796

24. Sarikaya H, Yilmaz M, Michael N, Miserez AR, Steinmann B, Baumgartner RW (2012) Zurich Fabry study-prevalence of Fabry disease in young patients with first cryptogenic ischaemic stroke or TIA. Eur J Neurol 19:1421-1426

25. Wozniak MA, Kittner SJ, Tuhrim S, Cole JW, Stern B, Dobbins $M$ et al (2010) Frequency of unrecognized Fabry disease among young European-American and African-American men with first ischemic stroke. Stroke 41:78-81
26. Bang OY, Ovbiagele B, Kim JS (2014) Evaluation of cryptogenic stroke with advanced diagnostic techniques. Stroke 45:1186-1194

27. Kappelle LJ, Adams HP Jr, Heffner ML, Torner JC, Gomez F, Biller J (1994) Prognosis of young adults with ischemic stroke. A long-term follow-up study assessing recurrent vascular events and functional outcome in the Iowa Registry of Stroke in Young Adults. Stroke 25:1360-1365

28. Neau JP, Ingrand P, Mouille-Brachet C, Rosier MP, Couderq C, Alvarez A et al (1998) Functional recovery and social outcome after cerebral infarction in young adults. Cerebrovasc Dis 8:296-302

29. Putaala J, Liebkind R, Gordin D, Thorn LM, Haapaniemi E, Forsblom C et al (2011) Diabetes mellitus and ischemic stroke in the young: clinical features and long-term prognosis. Neurology 76:1831-1837

30. Rutten-Jacobs LC, Maaijwee NA, Arntz RM, Schoonderwaldt HC, Dorresteijn LD, van der Vlugt MJ et al (2013) Long-term risk of recurrent vascular events after young stroke: the FUTURE study. Ann Neurol 74:592-601

31. Pezzini A, Grassi M, Lodigiani C, Patella R, Gandolfo C, Zini A et al (2014) Predictors of long-term recurrent vascular events after ischemic stroke at young age: the Italian Project on Stroke in Young Adults. Circulation 129:1668-1676 